\title{
Remote controlled gate controller using a GSM network and Arduino platform
}

\author{
Martin Pospisilik ${ }^{1, a}$, Tomas Smekal ${ }^{1}$, Milan Adamek $^{1}$, Petr Neumann $^{1}$ and Tomas Dulik ${ }^{1}$ \\ ${ }^{1}$ Faculty of Applied Informatics, Tomas Bata University in Zlin, Nad Stranemi 4511, 76005 Zlin, Czech Republic
}

\begin{abstract}
Most remote controllers for entrance gates operate on free frequencies 433 or $868 \mathrm{MHz}$. However, this technology limits the user comfort, as it is usually not common that bi-directional communication is established. A higher comfort of controlling the entrance gates can be achieved by employing the GSM network for transmission of commands and messages between the gate controller and the user. In this case, only a conventional GSM cellular phone is needed to control the gate. A description of such a controller based on the GSM module and Arduino controller is provided in this paper.
\end{abstract}

\section{Introduction}

Not only in Europe, the GSM (Global System for Mobile Communication) is widely spread, allowing to transfer speech signals but supporting data transfers as well. The most common devices to be connected to this network are cellular phones. On the other hand, there exist other devices that embody the interface needed to establish the connection. The broadest possibilities are then given by standalone GSM modules that are designed to be embedded into arbitrary devices. In this work, GSM shield based on SIM900 chip has been used.

\subsection{GSM network}

The block diagram of the GSM network is depicted in figure 1 . The description of the abbreviations is provided in table 1 .

The brief description of the operation of the GSM network is as follows: The mobile station is always identified by its IMEI (International Mobile Equipment Number) and its user is identified by his SIM (Subscriber Identification Module). By means of a radio connection it is connected to the system of base stations (BSS). The BSS is connected to the Network Switching Subsystem (NSS). The BTS stations of the BSS are spread around the covered area, creating a cellular system of transceivers. While the BSS is responsible for maintaining the connection between the mobile stations and the network, the NSS is responsible for authorization of the devices connected to the network (using HLR, AUC and VLR) and for switching the data in the communication network. The service of the whole system is then provided by the Operational and Support Subsystem (OSS).

\subsubsection{Data transfers in GSM network}

The development of the GSM network has been launched in 1982. Although it was intended to be used for speech transfers, the packetizing of the digital speech signal allowed to extend its functionality for transferring of general data. In 2000 the standard GPRS (General Packet Radio Service) has been implemented, mainly to allow more comfortable connection of the mobile stations to the Internet.

Table 1. Description on abbreviations depicted in figure 1.

\begin{tabular}{|c|c|}
\hline Abbreviation & Desctiption \\
\hline MS & Mobile station \\
\hline BTS & Base transceiver station \\
\hline BSC & Base station controller \\
\hline TC & Transcoder \\
\hline BSS & Base station subsystem \\
\hline NSS & Mobile switching centre \\
\hline MSC & Gateway mobile switching centre \\
\hline GMSC & Visitor location register \\
\hline VLR & Signalling system 7 \\
\hline SS7 & Interworking functionality \\
\hline IWF & Equipment identity register \\
\hline EIR & Home location register \\
\hline HLR & Authentication centre \\
\hline AUC & SMS centre \\
\hline SMSC & Public switched telephone network \\
\hline PSTN & Integrated services digital network \\
\hline ISDN & Operation subsystem \\
\hline OSS & Operation and maintenance centre \\
\hline OMC & Networking management centre \\
\hline NMC & Administrative centre \\
\hline ADC & \\
\hline
\end{tabular}

\footnotetext{
a Corresponding author: pospisilik@fai.utb.cz
} 
The GPRS system is designed to use the already set system of radio channels, but it treats the physical layer of the system in a different way. The implementation of the GPRS system in GSM networks is depicted in figure 2. As depicted, the implementation of the GPRS system to the GSM network consisted in adding several functionalities most of which are based on software. The Base Station Controllers must have been equipped with

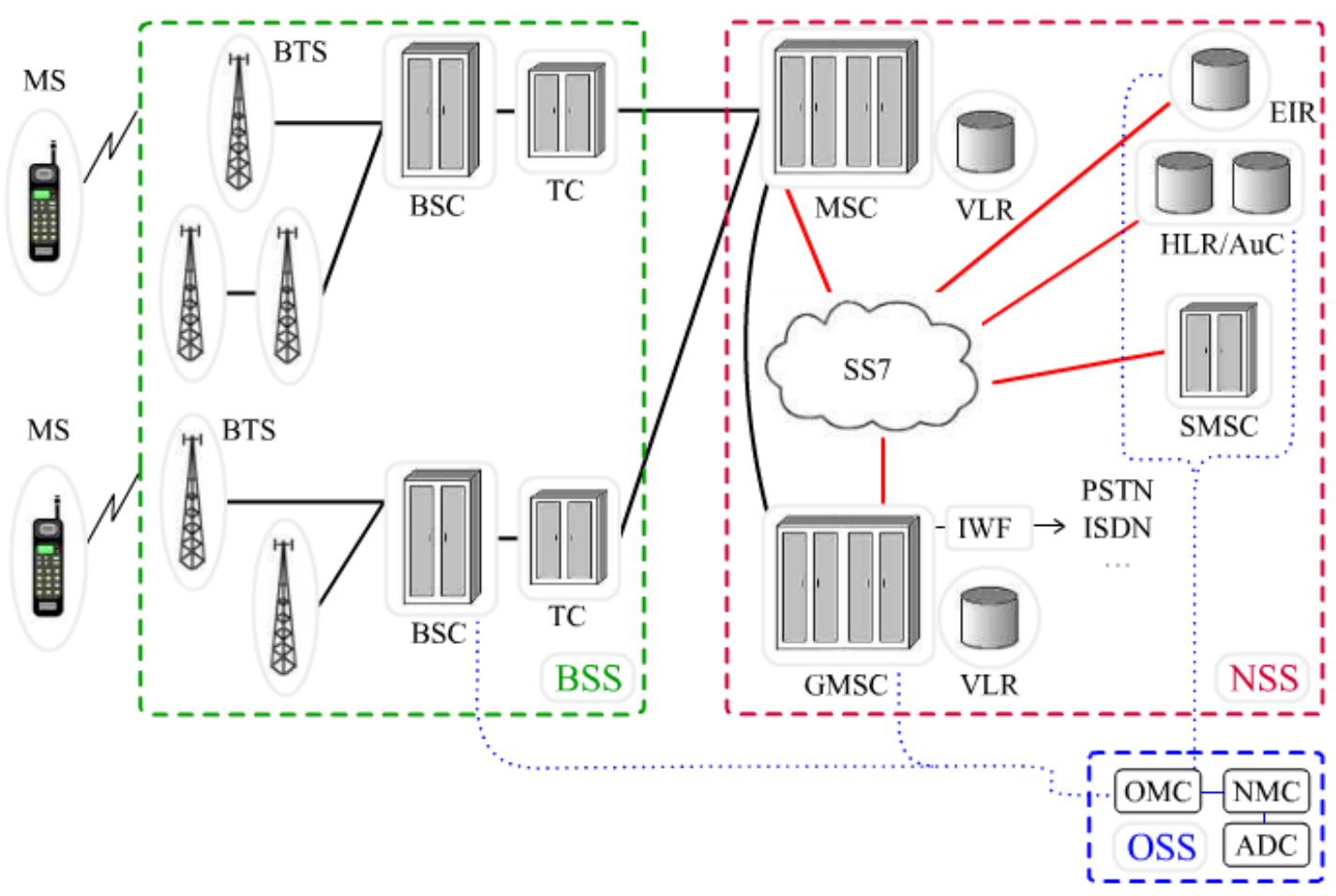

Figure 1. GSM network structure [4].

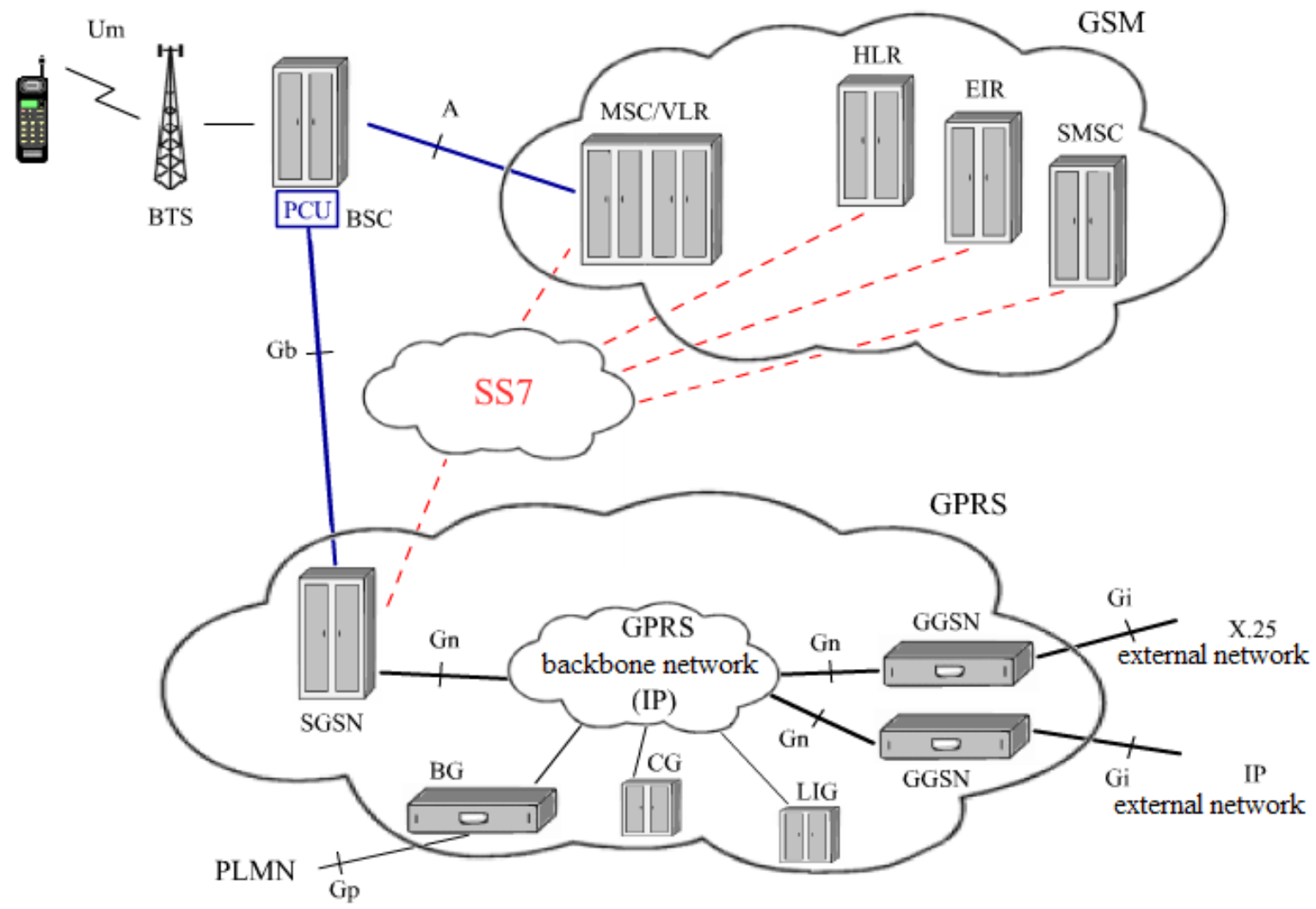

Figure 2. Implementation of GPRS system to the GSM network [4]. 
the hardware modules called PCU (Packet Controller Unit) that provided interface to the GPRS system. On the side of the GPRS, the interface is provided by SGSN (Servicing GPRS Support Node) that acts similar to MSC (Mobile Switching Centre). The signalization and controlling is provided also by the SS7 signalization protocol and from the GSM network, the following parts are used to identify the user and establish a communication with his mobile station: HLR, EIR, SMSC. The interface between the GPRS network and the networks based on different protocols (IP, X.25, etc.) is then provided by GGSN (Gateway GPRS Support Node). The system also incorporates the following units: $B G$ (Border Gateway) that enables connection to other networks (roaming), CG (Charging Gateway) that stores and processes information on the amount of transferred data and the LIG (Legal Interception Gateway) that enables interception of the data transfers by the authorities (applied in several countries).

\subsubsection{Security in GSM network}

The level of security of the services provided by the GSM network is limited by several aspects. Generally, the authentication of the device is processed by means of a pre-shared key and response to the request. The communication between the base station and the user's mobile station may be encrypted. The authentication of the user is performed by means of his SIM card, PIN (Personal Identification Number) and PUK (PIN Unlocking Key). All devices are also given their unique IMEI number (International Mobile Equipment Identity) that is stored in both the mobile device and the EIR (see Table 1). Several encryption methods are applied to the data transferred by the GPRS. The details can be found in [3]

\subsection{Remotely controlled gates}

The remotely constructed gates are usually based on one of the following constructions:

- Rail based,

- Self-supporting.

Both of these types are usually equipped with unified actuators that enable opening and closing of the gates together with supplementary functions as giving the information on the position and/or movement of the gate, self-diagnostics, etc. The area of the gate's range is monitored by sensors detecting obstacles that could collide with the gate when opening or closing. Usually the sensors based on infrared rays.

The example of the construction of the remotely controlled gate is depicted in Fig. 3.

The conventional remote controllers operate at the frequency of 433 or $868 \mathrm{MHz}$ respectively. The transceivers are distinguished by the unique code. Three types of authentication can be applied:

- Fixed code; the receiver and the transmitter use always the same code,
- Floating code; every time the transmitter is required to send a command, it uses different code. The receiver never accepts the same code again,

- Floating code with Antiscan; if the aggressor tries to guess the code, the receiver is blocked.

Application of controllers employing the GSM network can widen the functionality in the following aspects:

- A list of numbers being accepted can be set at the receiver.

- There is a possibility of bidirectional data transfers and sending messages to the user, for example when the gate is violently opened.

- The communication with the gate's controller can be processed easily by means of the user's cellular phone. The example of this solution can be found in [11].

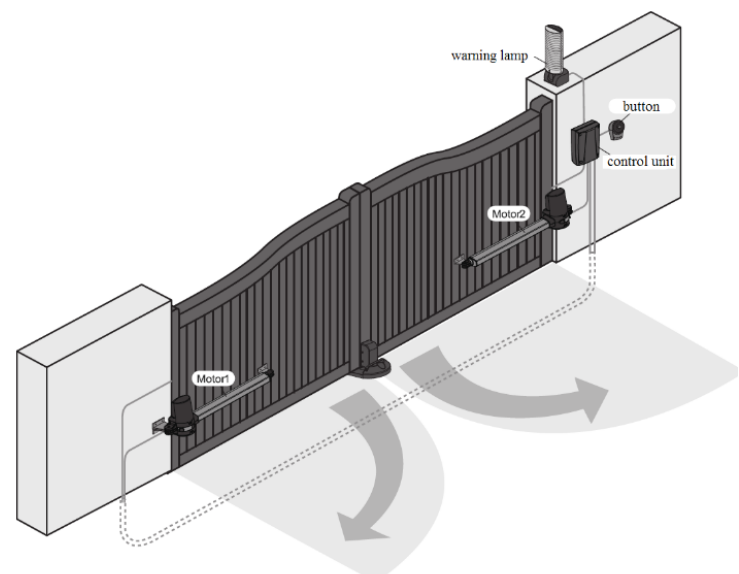

Figure 3. Example of construction of a remotely controlled gate with two drivers [5].

\subsection{Arduino}

Arduino is an open-source project that started in 2005 in Interaction Design Institute as a low cost development kit for applications with microcontrollers by Atmel. It is a physical platform with a microcontroller and several peripherals that is programmed by means of a simple development environment. Modular construction allows to connect expansion boards, called also as "shields". When programming, also the language $\mathrm{C}++$ can be applied. More information on Arduino project can be found in [6].

For the purposes of this project, the Arduino model UNO has been selected. It is based on the microcontroller Atmel ATmega328p. It is equipped with $14 \mathrm{I} / \mathrm{O}$ pins. Some of these pins support PWM, analog inputs and/or connecting of a $16 \mathrm{MHz}$ crystal. Using the shields, the following peripherals can be embedded in the unit: Ethernet, Bluetooth, LCD display, and, not least, the GSM module.

The parameters of Arduino Uno are provided in the table 2 . 
Table 2. Parameters of Arduino Uno.

\begin{tabular}{|l|l|}
\hline Parameter & Value \\
\hline Input voltage (operational) & $7-12 \mathrm{~V}$ \\
\hline Input voltage (limit) & $6-20 \mathrm{~V}$ \\
\hline Digital I/O pins & 14 \\
\hline Analog inputs & 6 \\
\hline Maximum current per a pin & $40 \mathrm{~mA}$ \\
\hline Flash memory & $32 \mathrm{kB}$ \\
\hline SRAM & $2 \mathrm{kB}$ \\
\hline EEPROM & $1 \mathrm{kB}$ \\
\hline Clock & $16 \mathrm{MHz}$ \\
\hline Dimensions (flat plane) & $68.6 \mathrm{x} 53.4 \mathrm{~mm}$ \\
\hline Height & $25 \mathrm{~g}$ \\
\hline
\end{tabular}

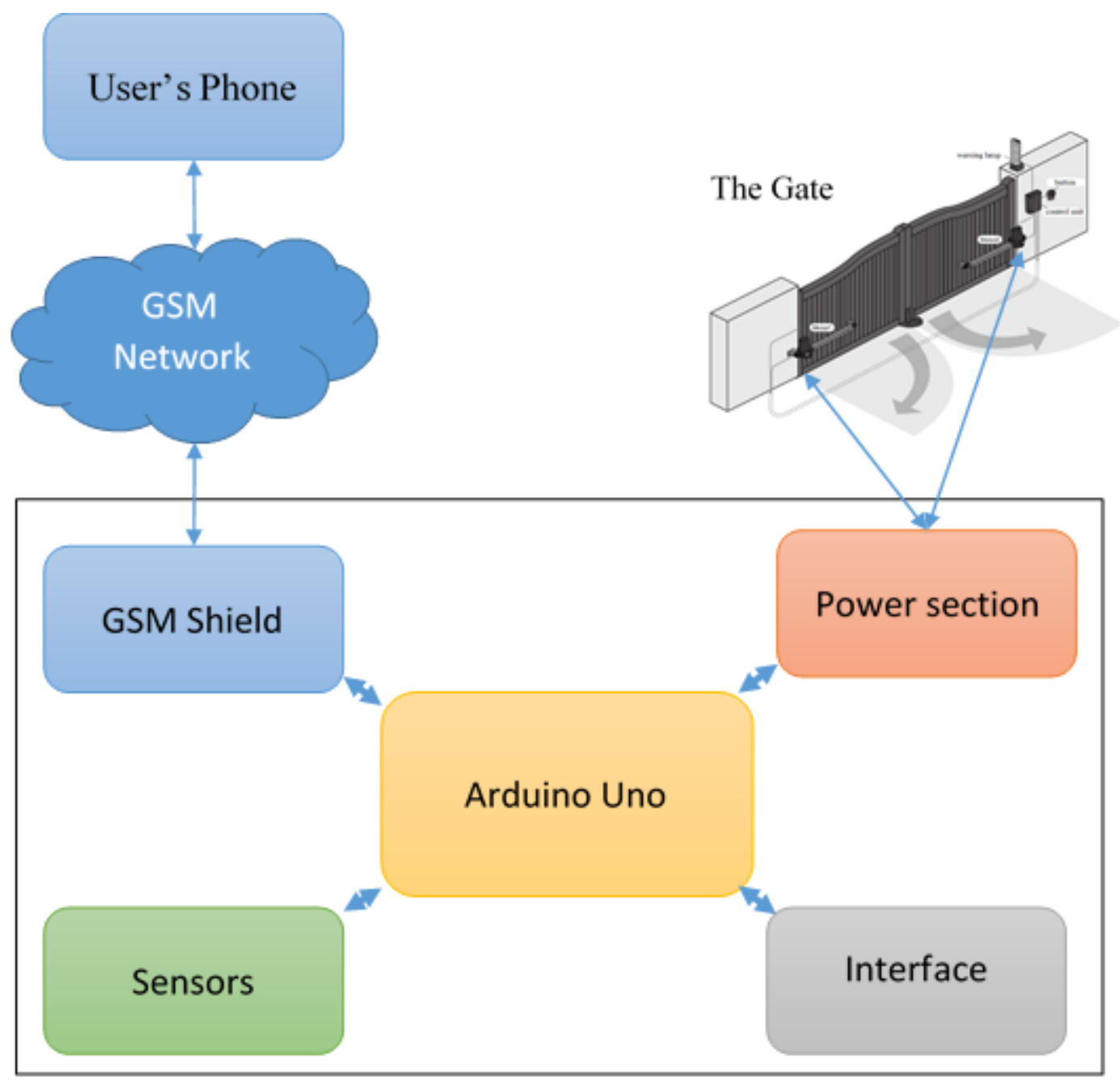

\subsection{GSM shield}

The GSM shield applicable for Arduino Uno that was used in this project, is based on the controller SIM900 by SIMCOM. It supports GSM/GPRS standards and operates at the frequencies of $850,900,1,800$ and 1,900 $\mathrm{MHz}$. The shield can be connected directly to the board of Adruino Uno by set of pins mounted at the bottom of the printed circuit board.

More information can be found in [7].

\section{Project description}

The hereby described project consists in development of a low-cost remote controlled driver for opening and closing of the gates. For the development, the above mentioned development kit Arduino Uno equipped with a GSM shield has been used. However, it was necessary to create another shield to provide interface between the controlling unit and the motors of the gate's driver unit.

The block diagram of the project solution is depicted in figure 4. The main development work consisted in making the Power section shield hardware, creating the software for the Arduino Uno board and making a model of the gate being controlled by the developed unit.

Figure 4. Concept of the remote controller using GSM network. 


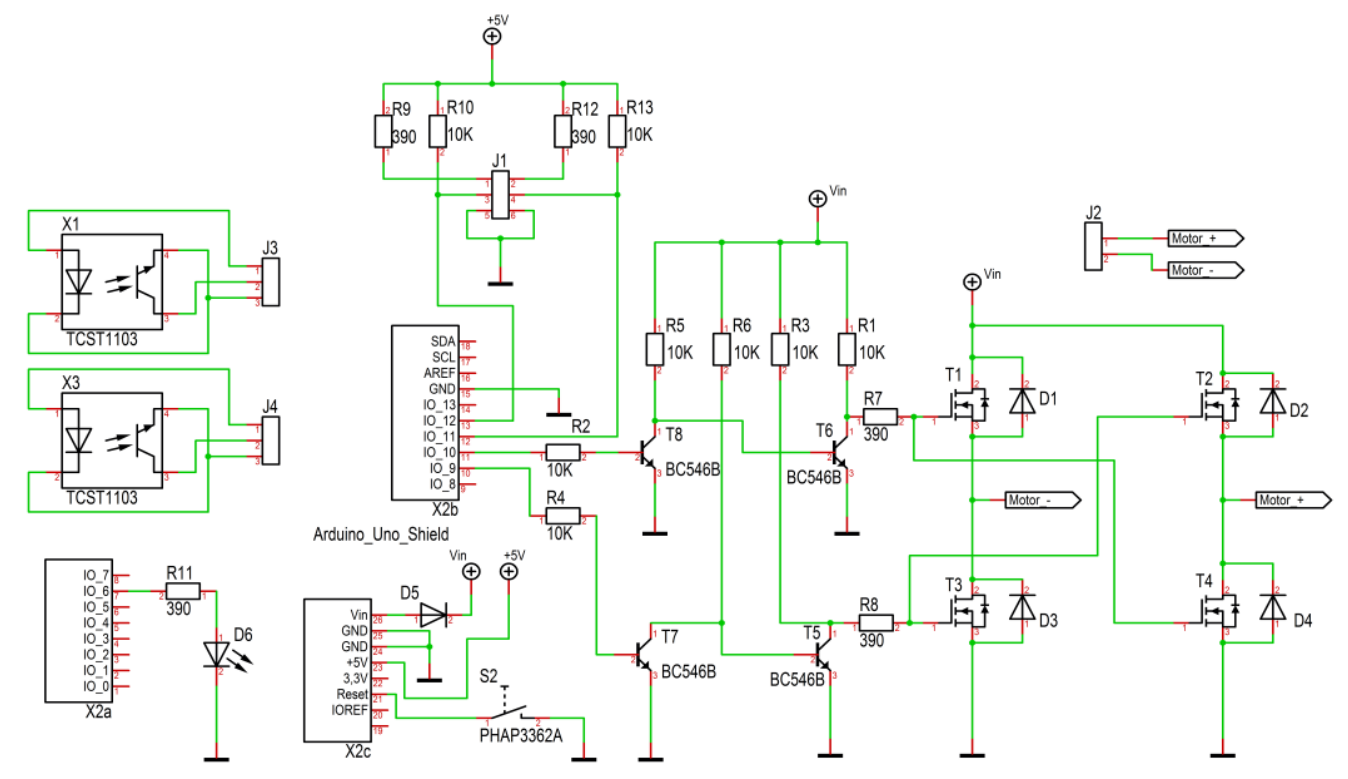

Figure 5. Connection diagram of the Power section shield [5].

\section{Implementation}

Within this chapter, hardware and software implementation is described.

\subsection{Hardware}

As noted above, as the Arduino Unit and its GSM shield were bought as the submodules, the another shield with the Power section had to be made in that way so it fit the Arduino Unit PCB directly by means of the connectors. Moreover, separated optical sensors were also created to check the obstacles in the area of the gate.

The connection diagram of the Power section shield is depicted in figure 5. The shield can be stacked to the units by means of the connectors X2a, X2b and X2c. The power section itself consists of a H-bridge, that is created by means of transistors $\mathrm{T} 1$ to $\mathrm{T} 4$ and the drivers of these transistors, that are made with transistors T5 to T8. The bridge is driven directly from the Arduino unit, using a PWM modulation that enables the possibility of controlling the speed of the gate's propulsion. The position of the gate is monitored by a pair of photocells that are connected to the connector J1. In the figure 5 the connection of these photocells is also depicted in the form of standalone circuits. The photocells employ TCST1103 modules that are marked as X1 or X3 in the diagram. Because the power consumption of the model of the gate is not high, the power supply to the Power unit is taken directly from the power supply network of the Arduino unit. The protection diode D5 was added to suppress the voltage ripples generated by the driven motor that could get back to the Arduino unit. If the output current of the driver is insufficient, more powerful $\mathrm{H}$-bridge can be connected to the power output of the shield. The power output is conducted through the connector $\mathrm{J} 2$.

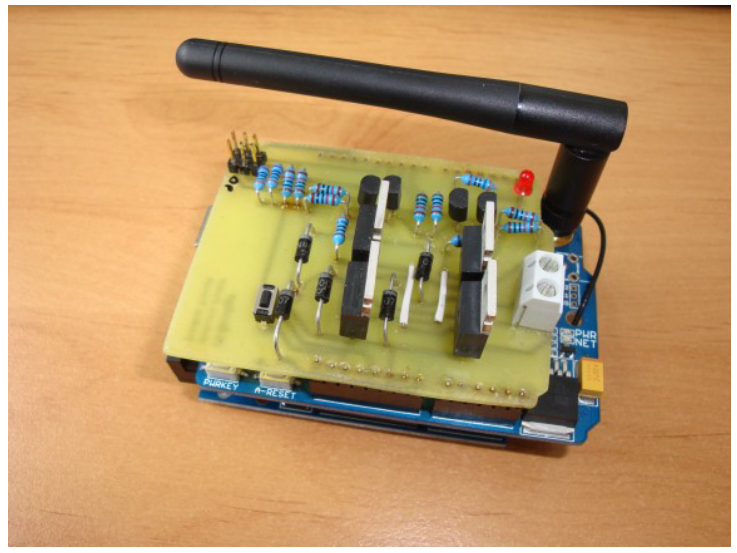

Figure 6. Completed gated controller [5].

The stack of three printed circuit boards can be seen in the figure 6. The PCBs are (from the top):

1. Power section shield,

2. GSM/GPRS shield,

3. Adruino Uno.

\subsection{Software}

The software implemented in the Arduino unit must distinguish between the following states:

- Opened - the gate is opened, the motor is off, the stop sensor of this position gives logical 1 at its output,

- Closed - the gate is closed, the motor is off, the stop sensor of this position gives logical 1 at its output, 
- Opening - both stop sensors give logical 0 at their outputs and the motor is turning in the direction to open the gate,

- Closing - the stop sensors give logical 0 at their outputs and the motor is turning in the direction to close the gate,

- Error - occurs when both stop sensors give the logical 1 at their outputs or both sensors give the logical 0 at their outputs and the motor is off.

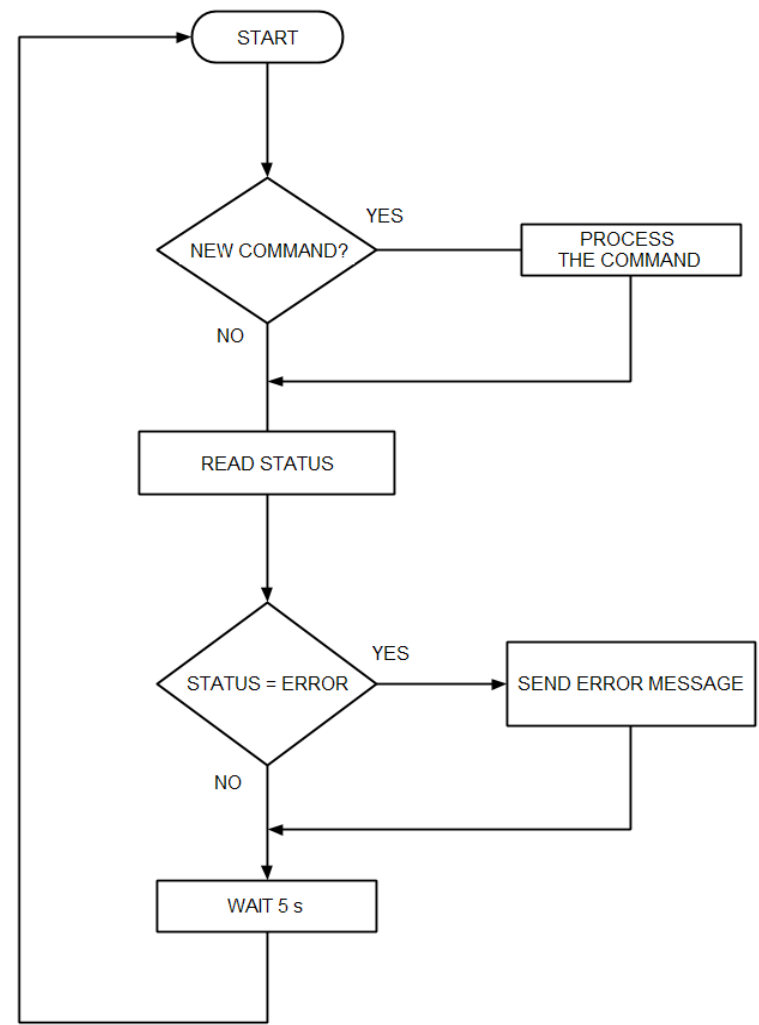

Figure 7. Flowchart of the software loop [5].

In this project, communication by means of SMS has been chosen. Therefore, the software must support the following commands that are sent via SMS:

- Open - calls the method gate.Open() that process opening of the gate,

- Close - calls the method gate.Close() that process closing of the gate,

- Slower - this command is determined by the set of characters "_" entered consecutively. By this command the speed of the gate's movement is decreased according to the number of the characters sent. The information on required speed of the gate is stored in the memory.

- Faster - this command is determined by the set of characters " + " entered consecutively. By this command the speed of the gate's movement is increased according to the number of the characters sent. The information on required speed of the gate is stored in the memory.

- Status - this command calls the method gate.Status() the result of which is to send the information on the status of the gate to the user by means of the SMS.
The application is repeated in a loop the diagram of which is depicted in the figure 7.

The Arduino's producer provides an open-source library GSM_GPRS_GPS_Shield that includes a set of classes for treating the GSM modules based on the controllers SIM900 and/or SIM908.

\section{Conclusions}

This paper provides a description on construction of the controller of the remotely controlled entrance gates that can be commanded via the user's cellular phone, employing data transfers in GSM network. In the framework of this project the functional sample was created on the basis of the development kit Arduino Uno and two embedded shields. The picture of the completed unit can be found in the figure 6 .

The created sample is fully operational, providing large possibilities to implement expansion functionalities. For example, as the hardware supports connection of additional units, a logging unit can be added to monitor all attempts to control the driver. Another option is adding of the LAN unit that would allow to connect the driver to the LAN network.

\section{Acknowledgements}

This work was supported by the Ministry of Education, Youth and Sports of the Czech Republic within The National Sustainability Programme Project No. LO1303 (MSMT-7778/2014) and also by The European Regional Development Fund under the project CEBIA-Tech No. CZ.1.05/2.1.00/03.0089.

\section{References}

1. S. F. Barrett, Arduino microcontroller: processing for everyone! (Morgan, 2012, ISBN 978-160-8458592)

2. B. Evans, Beginning Arduino programming: writing code for the most popular microcontroller board in the world (Apress, 2011, ISBN 978-1-4302-3777-8)

3. T. Halonen, J. Melero, J. Romero, GSM, GPRS and EDGE Performance: Evolution Towards 3G/UMTS, 2nd Edition (John Wiley, 2003, ISBN 04-708-66942)

4. Richtr, T., Basic structure of GSM network (Online: URL http://tomas.richtr.cz/mobil/gsm-strukt.htm)

5. Smékal, T., The Remote control of gates using a GSM unit (Diploma Thesis, 2015, Supervizor: M. Pospisilik)

6. Arduino - ArduinoBoardUno. (Online: URL http://www.arduino.cc/en/Main/ArduinoBoardUno)

7. SIM900 Quad-Band GPRS shield with Micro SD card slot - Epalsite Wiki (Online: URL http://wiki.epalsite.com/index.php?title=SIM900_Q uadBand_GPRS_shield_with_Micro_SD_card_slot) 